\title{
Behavior Measurement, Analysis, and Regime Classification in Car Following
}

\author{
Xiaoliang Ma, Student Member, IEEE, and Ingmar Andréasson
}

\begin{abstract}
This paper first reports a data acquisition method that the authors used in a project on modeling driver behavior for microscopic traffic simulations. An advanced instrumented vehicle was employed to collect driver-behavior data, mainly carfollowing and lane-changing patterns, on Swedish roads. To eliminate the measurement noise in acquired car-following patterns, the Kalman smoothing algorithm was applied to the state-space model of the physical states (acceleration, speed, and position) of both instrumented and tracked vehicles. The denoised driving patterns were used in the analysis of driver properties in the car-following stage. For further modeling of car-following behavior, we developed and implemented a consolidated fuzzy clustering algorithm to classify different car-following regimes from the preprocessed data. The algorithm considers time continuity of collected driver-behavior patterns and can be more reliably applied in the classification of continuous car-following regimes when the classical fuzzy $\mathrm{C}$-means algorithm gives unclear results.
\end{abstract}

Index Terms-Car-following regime classification, driver behavior, fuzzy clustering algorithms, instrumented vehicle, Kalman smoothing.

\section{INTRODUCTION}

$\mathbf{I}$ NTELLIGENT transportation systems (ITS) is a multidisciplinary area with its focus on incorporating up-to-date information technologies of all kinds in the field of transportation. Recent advances in intelligent and automated vehicles, and continuous improvements of information-based road infrastructures, have propelled research to understand their interactions and to evaluate them on a large scale. This evaluation will, in return, provide valuable feedback not only on how to improve the road-based information and control systems but on how to design vehicle-based intelligent systems. Simulation has become a cost-effective option for the evaluation of infrastructure improvements, on-road traffic management systems, and invehicle driver support systems due to the fast evolution of computational modeling techniques. Moreover, a rapid development of sophisticated microscopic simulation models over the past decade has led to a tide of applications of microsimulation in transportation engineering.

Manuscript received January 31, 2006; revised May 31, 2006, June 12, 2006, and July 17, 2006. This work was supported by the SIMLAB project at the Center for Traffic Simulation Research (CTR), Royal Institute of Technology (KTH), Sweden. This work was also supported in part by the Division of Transportation and Logistics (TOL), Department of Transport and Economics, $\mathrm{KTH}$. The Associate Editor for this paper was N. Zheng.

The authors are with the Center for Traffic Simulation Research (CTR), Royal Institute of Technology, 10044 Stockholm, Sweden (e-mail: liang@ ctr.kth.se; ingmar@ctr.kth.se).

Color versions of one or more of the figures in this paper are available online at http://ieeexplore.iee.org.

Digital Object Identifier 10.1109/TITS.2006.883111

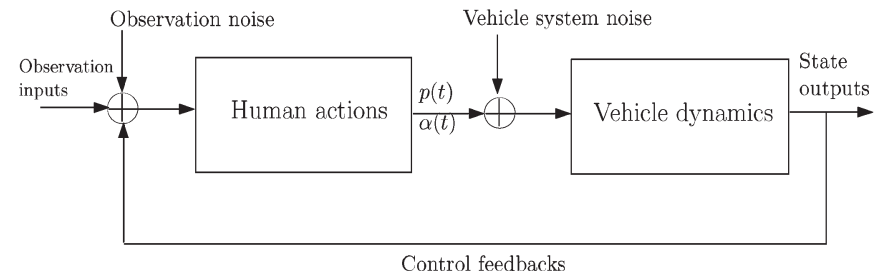

Fig. 1. Structure of a driver-vehicle-unit model: $p(t)$ and $\alpha(t)$ are driver action outputs of pedal (brake) pressure and steering angle.

Humans play an essential role in the operation and control of human-machine systems such as driving a car. Modeling driver behavior has transferred human skills to intelligent systems, e.g., the adaptive cruise control (ACC) system, intelligent speed adaption (ISA) system, and autonomous vehicles. Human driving models are also indispensable for the performance evaluation of transportation systems. With advances in emerging vehicle-based ITS technologies, it becomes even more important to understand the normative behavior response of drivers and changes under new systems. Based on Rasmussen's human-machine model [28], driver behavior can also be separated into a hierarchical structure with three levels: the strategic, tactical, and operational level. At the highest or strategic level, goals of each driver are determined, and a route is planned based on these goals. The lowest operational level reflects the real actions of drivers, e.g., steering, pressing pedal, and gearing. In the middle tactical level, certain maneuvers are selected to achieve short-term objectives, e.g., interactions with other road users and road infrastructures. The behavior at this level is dominated by the most recent situations but is also influenced by drivers' goals at the higher level. To develop microscopic traffic simulation of high fidelity, researchers are often interested in imitating human's real driving behavior at a tactical level. That is, without describing the detailed driver actions, driver-vehicle units (DVUs) in the simulation are modeled to replicate their states in reality, i.e., the profiles of vehicle position, velocity, acceleration, and steering angle. Fig. 1 shows the model structure of a DVU in which the detailed driver actions become internal.

Car following and lane changing are two crucial tactical-level models for a microscopic simulation system. Car following describes the longitudinal action of a driver when he follows another car and tries to maintain a safe distance to the leading car. Lane changing illustrates the lateral behavior of a driver between two lanes of a multilane road when he intends to escape from possible conflicts with other cars in the same lane. Initial research on driver behavior can be traced back to the 


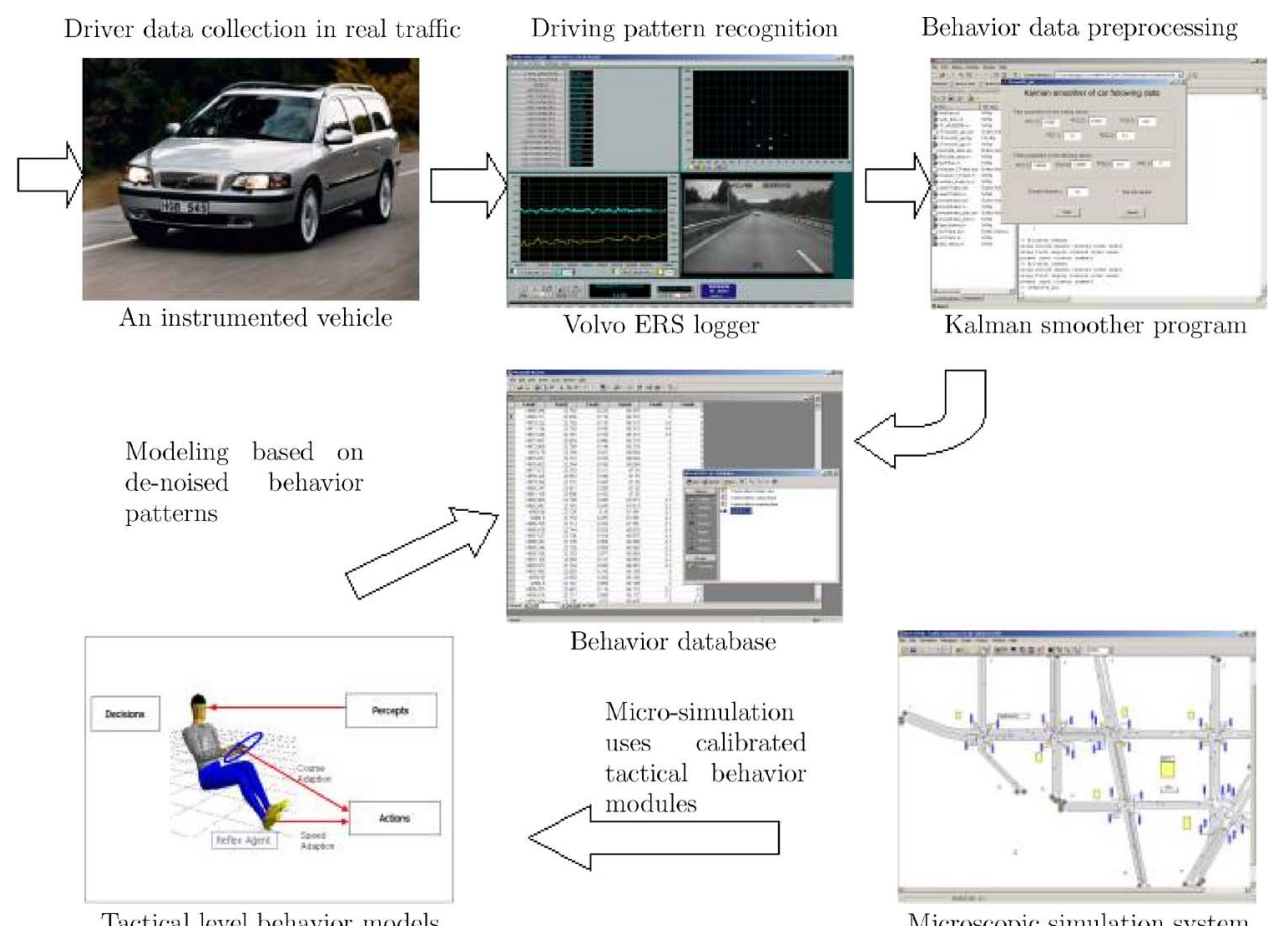

Fig. 2. Processes to obtain driver-behavior patterns from instrumented-vehicle-based observation and applications of the data in the calibration and development of models in a microsimulation environment.

1950s when the basic traffic flow theory was developed and carfollowing model became a crucial element. The original idea was to design a mathematical form being able to describe the car-following behavior exactly and, meanwhile, to replicate macroscopic relations (e.g., flow density) appropriately. The well-known nonlinear General Motor car-following model, also called the GHR model, was introduced by Gazis et al. in 1961 [10] based on the early study of the stimulus-response-type carfollowing models [7]. It took a mathematical form of delay-type differential equations as follows:

$$
a_{n}\left(t+\tau_{n}\right)=\alpha \frac{v_{n}\left(t+\tau_{n}\right)^{\beta}}{D_{n}(t)^{\gamma}}\left[v_{n-1}(t)-v_{n}(t)\right]
$$

where $s(t), v(t)$, and $a(t)$ are the position, speed, and acceleration of the vehicles, $D_{n}(t)=s_{n-1}(t)-s_{n}(t)-L_{n-1}$ is the distance headway, and $L_{n-1}$ is the length of the leading vehicle. $\tau_{n}$ is the reaction time of the driver and always assumed to be a fixed value for a certain driver $n ; \alpha, \beta$, and $\gamma$ are constant parameters. An intuitive hypothesis of the model assumes that the follower's acceleration is proportional to the speed difference term $d v(t)=v_{n-1}(t)-v_{n}(t)$ and the exponent of its own speed but being inversely proportional to that of distance headway $D_{n}(t)$. The speed difference part at the right-hand side has always been translated as the "stimulus term," while the fraction between exponent of following speed and that of distance headway is called "sensitivity term." Besides differential equations, many other approaches were proposed such as the psychophysical models [21], fuzzy inference systems [6], control-based models [2], and so on. Although more factors [19] might be involved in the follower's decision in the real traffic environment, the variables above show strong correlation to the driver's decision and are relatively easy to observe using modern equipment.

Previously, scarcity of accurate and consistent data had been one of the main barriers in the development of realistic driverbehavior models. Later on, test tracks and static laboratory simulators were used as major tools to obtain human behavior data under different driving tasks. With the increasing availability of vehicle-based ITS technologies, instrumented cars capable of acquiring data in real traffic environments have become an advanced means in a variety of research applications, e.g., in [4]. As described in Fig. 2, we intend in this research project to build a series of tools to obtain clear and consistent driver-behavior patterns and formulate a database from the instrumented-vehicle-based data acquisition. The behavior patterns have been further applied to calibrate established tactical driver models and to develop new models using data-mining approaches. All models are intended to be implemented in and integrated into a microsimulation system (e.g., traffic performance on major arterial (TPMA) [20] and [23]) in the near future. Although both car-following and lanechanging processes were observed in our experiment, current studies have been mainly focused on car-following behavior.

The remainder of this paper is organized as follows: In the second section, the driver-behavior data-collection method using an instrumented vehicle is introduced; the third section demonstrates the principle and practice of the noise cancellation process by Kalman smoothing; meanwhile, the denoised carfollowing data was used in the identification of driver properties; the fourth section introduces a fuzzy clustering algorithm, which can classify car-following regimes in the data surveying procedure. 


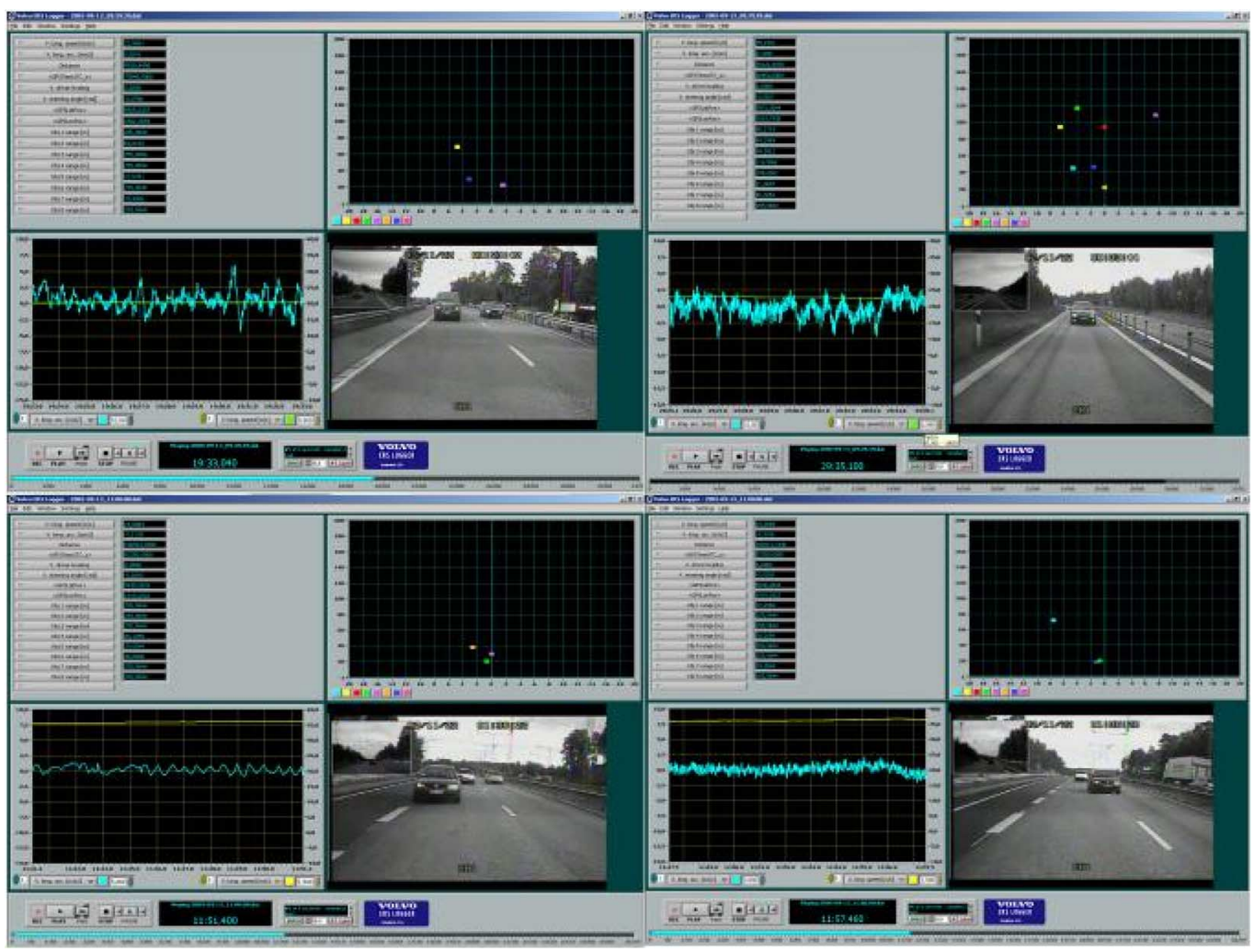

Fig. 3. Volvo ERS logger interface with captured unenforced car following (upper left), enforced car following (upper right), cut-in (lower left), and lane changing (lower right).

\section{Method For Driver-Behavior Data AcQuisition}

An instrumented car (Volvo V70 with a manual five-speed gearbox and 2.4-L engine) in Fig. 2 developed by Volvo Technology was employed in our experiment on driver-behavior data acquisition. A data-collection method based on a similar equipped vehicle had been examined in our early studies on driver behavior of ISA vehicles [26] and [24]. However, both the hardware and software in this vehicle were recently updated, and the experiment was more carefully designed for the observation of tactical driver behavior of other vehicles in the real traffic flow. This instrumented vehicle was equipped with a GPS-based navigation system and an advanced on-board trip computer. The following information can be recorded:

1) GPS-based information including coordinates, GPS speed, etc.;

2) travel time, speed and distance, and fuel economy;

3) longitudinal and lateral acceleration rates;

4) yaw rate and steering wheel angle;

5) applied brake-pedal pressure, engine speed, etc.

Connecting with the main computer, a pair of two-dimensional scanning lidar sensors were set up to observe, at most, four objects in both front and rear sides and to measure their relative range, relative range rate, relative acceleration, angle, lateral speed, and so on. Two video cameras were also installed to record real-time situations in both directions. The Volvo ERS software running on a portable laptop computer can log all information infused from those equipment at a maximum frequency of 50 times per second in Hertz. Fig. 3 shows the interface of Volvo ERS software where runtime data can be analyzed together with the synchronal video recording and the car-following and lane-changing situations captured by the equipment. It is worth mentioning that the software has not been armed with the intelligence to recognize behavior patterns automatically regarding the complexity in the general road environment, though this might be one of our future research objectives. Hence, the driving patterns are mainly decided by us based on the synchronal video recording.

In the experiment, the behavior of random vehicles behind, especially followers observed by the lidar hiding at the rear side, is our main interest for the car-following study. The lidar sensor can continuously measure the range, relative range rate, and relative acceleration to the following vehicles driven by unknowns. Since one lidar was concealed behind, the drivers being observed at the rear side did not know that their driving behaviors were recorded. Therefore, we could obtain behavior patterns of a random population of subjects. Moreover, to give a certain variance of the driving style to our instrumented car, five employees at our institute drove this car in turn. Since we have one lidar sensor and a video camera in the front of the instrumented car, not only detailed actions but the car-following behavior of all five subjects were also logged in our database. These could be used in our future comparison study. For the car-following study, a multivariate time series can be obtained from the output file of the Volvo ERS software. The time series can be written as

$$
\mathbf{x}(t)=\left[\begin{array}{llllll}
s_{i}(t) & v_{i}(t) & a_{i}(t) & D(t) & d v(t) & d a(t)
\end{array}\right]^{\mathrm{T}}
$$



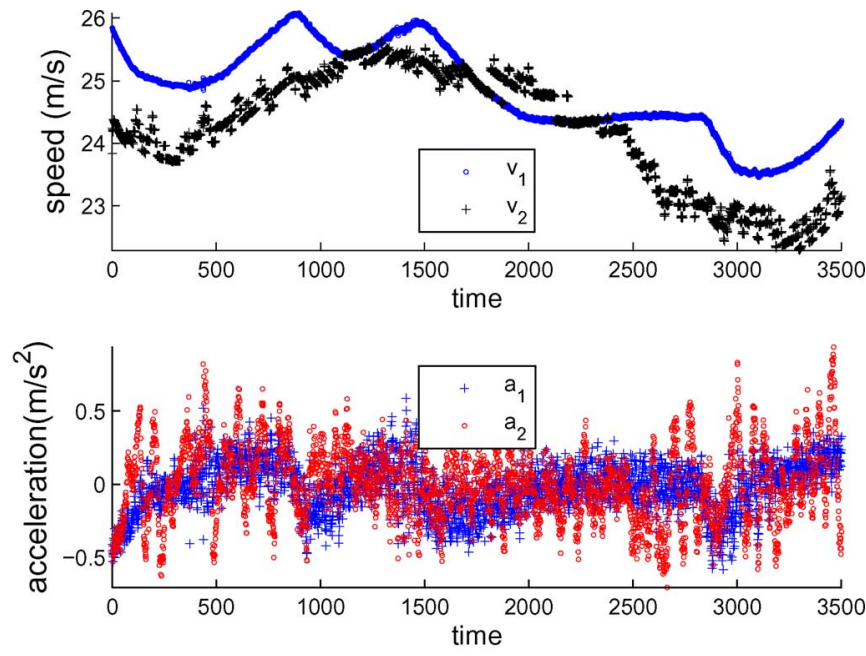

Fig. 4. Example plot of raw data outputs from the equipment of the instrumented vehicle: the speed profiles (upper) and the acceleration curves (lower) of two cars.

where $s_{i}(t), v_{i}(t)$, and $a_{i}(t)$ are travel distance, speed, and acceleration of the instrumented vehicle $i$, and $D(t), d v(t)$, and $d a(t)$ are relative distance, speed, and acceleration between the instrumented vehicle and the observed one. Therefore, the physical state of the observed vehicle $j$ at time $t$ can be derived by

$$
\left(\begin{array}{c}
s_{j}(t) \\
v_{j}(t) \\
a_{j}(t)
\end{array}\right)=\left(\begin{array}{c}
s_{i}(t)-D(t) \\
v_{i}(t)-d v(t) \\
a_{i}(t)-d a(t)
\end{array}\right) .
$$

All experimental runs were conducted in a $25-\mathrm{km}$-long section of the motorway E18, north of Stockholm City. To measure broader types of car-following behavior on the highway, the instrumented vehicle was employed to acquire data in both double-lane and single-lane road sections. In the test road section, there are mainly three speed limits: 70, 90, and $110 \mathrm{kmi} / \mathrm{h}$. In the double-lane roads, both car-following and lane-change behavior were observed. Since followers had opportunities to overtake us on double-lane roads, their behavior mostly belonged to unforced car following. In the singlelane road, many of the drivers with higher desired speed were obliged to follow us, and so, they often showed more aggressive following behavior, e.g., close following.

The measuring frequency adopted in our experiment is either 25 or 50 times per second in Hertz, which means that the time interval was either $h=0.04$ or $h=0.02 \mathrm{~s}$. This high measuring frequency should give enough resolution to our later studies on car following. However, the affordable lidar sensor can only continuously measure the range value on which the relative range rate and relative acceleration are approximated by taking numerical differentials. This means that the measuring noise of lidar sensors (1\%-5\% of the range) will be amplified when deriving its differentials. To alleviate this effect, the Volvo ERS software integrates an online adaptive linear filtering algorithm to give smoother readings of relative range and acceleration, though it results in a small delay in the time series. Fig. 4 shows the measurement output: the speed and acceleration of both the leading and following cars. From the pictures, we can tell, however, that the measured states of both cars, especially the observed car, show quite noisy patterns and are therefore not appropriate for direct application in behavior modeling. In the next section, we will introduce an offline smoothing algorithm, which can more efficiently cancel the noise from the measurement.

\section{Preprocessing of CAR-Following Data}

Whenever the state of a system must be estimated from noisy sensor information, some kind of state estimator is needed to extract the data from different sensors to produce an accurate state estimate of the true system. There are different methods of estimating the real signal or time series from the noisecorrupted signal or time series. Taking the moving average values or using a low-pass filter to deal with high-frequency noise are often used in data preprocessing in traffic engineering [30] and other fields. In fact, these methods can be generalized as filtering data series with a linear time invariant (LTI) filter [13]

$$
y(t)=\sum_{l=-q}^{q} \phi(l) x(t-l) .
$$

However, an LTI filter assumes that the data process is stationary and that the filter parameters are predetermined without optimization. These bring risks of losing information on the real signal. Wiener filtering, on the other hand, applies the linear filtering approach and minimizes the mean-square error, but it has limitations in its applicability to nonstationary and multivariate signals.

\section{A. State Space Model}

The Kalman filter [17] is an optimal linear filtering approach that can be treated with nonstationary data processes in an iterative way, and it is specially appropriate for the state-space model form

$$
\begin{aligned}
\mathbf{X}(t+1) & =F(t) \cdot \mathbf{X}(t)+G(t) \cdot \mathbf{V}(t) \\
\mathbf{Y}(t) & =H(t) \cdot \mathbf{X}(t)+\mathbf{W}(t)
\end{aligned}
$$

where $\mathbf{X}(t)$ is the state vector, and $\mathbf{Y}(t)$ is the measurement vector at time $t . F(t)$ is the state transition matrix, and $H(t)$ is the relation matrix between measurement and state vector. Here, we have not considered any control input in the state equation. Furthermore, the Kalman filtering approach has been extended to be treated with nonlinear systems using the (extended Kalman filter) EKF method [11], [29], in which the model is linearized so that the traditional linear equations can be applied. In this paper, however, we intend to apply the Kalman smoothing algorithm, which is an extension from the classical linear Kalman filter, to estimate the states of vehicles being tracked by our equipped car. Before introducing the smoothing algorithm, the state-space model needs to be formulated in advance. 


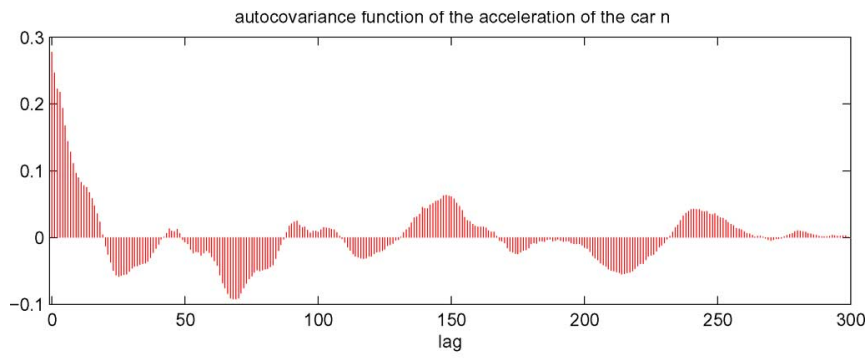

partial autocorrelation function of the acceleration of the car $n$

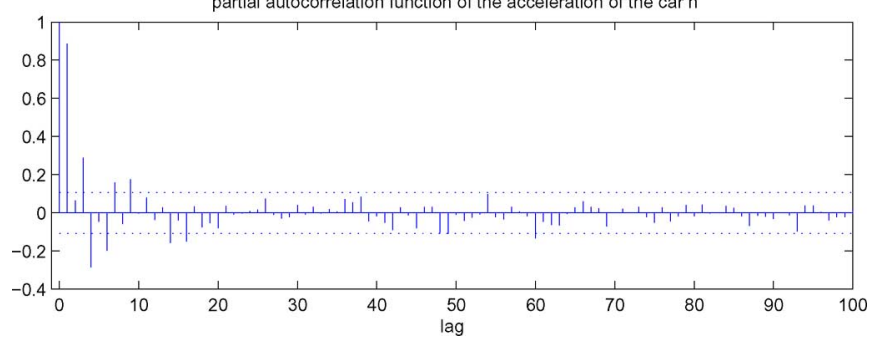

Fig. 5. Autocovariance and partial autocorrelation function of the acceleration of car $n$.

For a traveling car being tracked, it is natural to formulate a state space model according to the physical state relation as follows:

$$
\begin{aligned}
& s_{n}(t+1)=s_{n}(t)+v_{n}(t) h+\frac{1}{2} a_{n}(t) h^{2} \\
& v_{n}(t+1)=v_{n}(t)+a_{n}(t) h .
\end{aligned}
$$

Nevertheless, we need an equation on the acceleration to complete the state-space model. Referring to the autocovariance function $(\mathrm{ACF})$ and partial ACF of the noise corrupted time series of the acceleration in Fig. 5, we then find an evidently strong correlation between $a_{n}(t)$ and $a_{n}(t-1)$. According to the order selection approach of autoregressive (AR) models [5], it is suggested to represent the acceleration time series as the first-order AR model

$$
a_{n}(t+1)=\phi a_{n}(t)+\theta(t)
$$

where $\theta(t)$ is a white noise $\mathrm{WN}\left(0, \sigma^{2}\right)$. The time series $a_{n}(t)$ will be stationary $\operatorname{AR}(1)$ process as far as $|\phi|<1$. If the unit root exists, e.g., $\phi=1$, the time series will be a first-order ARI process. This model represents a random walk process in which acceleration at the next time interval equals acceleration at current time interval with an addition of a random noise term. In summary, the state space equation for our problem can be written as

$$
\begin{aligned}
\mathbf{X}(t+1) & =F \cdot \mathbf{X}(t)+\mathbf{V}(t) \\
\mathbf{Y}(t) & =H \cdot \mathbf{X}(t)+\mathbf{W}(t)
\end{aligned}
$$

where $\mathbf{X}(t)=\left[\begin{array}{lll}s_{n}(t) & v_{n}(t) & a_{n}(t)\end{array}\right]^{\mathrm{T}}, \quad \mathbf{V}(t)=\left[\begin{array}{lll}0 & 0 & \theta(t)\end{array}\right]^{\mathrm{T}}$, $\mathbf{Y}(t)=\left[\hat{s}_{n}(t) \hat{v}_{n}(t) \hat{a}_{n}(t)\right]^{\mathrm{T}}, H=I$, and

$$
F=\left(\begin{array}{ccc}
1 & h & h^{2} / 2 \\
0 & 1 & h \\
0 & 0 & \phi
\end{array}\right)
$$

It is worth mentioning that we present a general case fullorder physical states (position, speed, and acceleration) in the observation equation.

\section{B. Kalman Smoothing Algorithm}

Besides Kalman's famous paper, there are many other texts (such as [5], [11], [13], [14], and [18]) that describe the Kalman filtering algorithm and its extensions. Different notations have been adopted in their formulations. Here, we only summarize the discrete Kalman algorithms based on the texts and notations with which we are familiar.

In essence, the Kalman filter is a minimum mean square estimator (mmse) given the linear state space model on $\mathbf{X}(\mathbf{t})$ and the observation sequence $\mathbf{Y}(t)$. The basic Kalman filter as an estimator for the state space model of (3) can be derived as the following iterative algorithm:

$$
\begin{aligned}
\hat{\mathbf{X}}_{t \mid t-1} & =F_{t-1} \hat{\mathbf{X}}_{t-1 \mid t-1} \\
\Omega_{t \mid t-1} & =F_{t-1} \Omega_{t-1 \mid t-1} F_{t-1}^{\mathrm{T}}+G_{t-1} R_{t-1}^{V} G_{t-1}^{\mathrm{T}}
\end{aligned}
$$

and

$$
\begin{aligned}
\hat{\mathbf{X}}_{t \mid t} & =\hat{\mathbf{X}}_{t \mid t-1}+\Gamma_{t}\left(\mathbf{Y}_{t}-H_{t} \hat{\mathbf{X}}_{t \mid t-1}\right) \\
\Omega(t \mid t) & =\left(I-\Gamma_{t} H_{t}\right) \Omega_{t \mid t-1} \\
\Gamma_{t} & =\Omega_{t \mid t-1} H_{t}^{\mathrm{T}}\left[H_{t} \Omega_{t \mid t-1} H_{t}^{\mathrm{T}}+R_{t}^{W}\right]^{-1}
\end{aligned}
$$

where $\hat{\mathbf{X}}_{t \mid i}:=P_{i}\left(\mathbf{X}_{t}\right)$ defines the projection of $\mathbf{X}_{t}$ on the linear span space of $\mathbf{Y}^{i}:=\left\{\mathbf{Y}_{k} \mid k=0 \ldots i\right\}$ according to the orthogonal principle. $\Omega_{t \mid i}=E\left[\left(\mathbf{X}_{t}-\hat{\mathbf{X}}_{t \mid i}\right)\left(\mathbf{X}_{t}-\hat{\mathbf{X}}_{t \mid i}\right)^{\mathrm{T}}\right]$, where $i=$ $t-1, t$ represents the a priori and posterior error covariance matrices. Equation (7) illustrates the state update of the estimator, while (8) describes the measurement update of the estimator. It is worth mentioning that both the process noise $\mathbf{V}(t)$ and the measurement noise $\mathbf{W}(t)$ are assumed to be white, and hence, $R^{V}$ and $R^{W}$ are the autocovariance matrix of noise processes $\mathbf{V}(t)$ and $\mathbf{W}(t)$, respectively.

In the offline smoothing, the complete observation sequence $\mathbf{Y}_{0}, \ldots, \mathbf{Y}_{N}$ is known, and therefore, noncausal information can be used for the noise cancellation to get the optimal solution. The Kalman fixed interval smoothing algorithm, also called the Rauch-Tung-Striebel smoother [11], [12], was extended from the basic Kalman filter above using this principle. It also includes a backward filtering procedure as follows:

$$
\begin{aligned}
\hat{\mathbf{X}}_{t \mid N} & =\hat{\mathbf{X}}_{t \mid t}+\Lambda_{t}\left(\hat{\mathbf{X}}_{t+1 \mid N}-\hat{\mathbf{X}}_{t+1 \mid t}\right) \\
\Omega_{t \mid N} & =\Omega_{t \mid t}+\Lambda_{t}\left(\Omega_{t+1 \mid N}-\Omega_{t+1 \mid t}\right) \Lambda_{t}^{\mathrm{T}} \\
\Lambda_{t} & =\Omega_{t \mid t} F_{t}^{\mathrm{T}} \Omega_{t+1 \mid t}^{-1}
\end{aligned}
$$

where $\hat{\mathbf{X}}_{t \mid N}:=P\left(\mathbf{X}_{t} \mid \mathbf{Y}^{N}\right)$ is the estimation of $\mathbf{X}_{t}$, given the data sequence $\mathbf{Y}_{0} \cdots \mathbf{Y}_{N} ; \Omega_{t \mid N}=E\left[\left(\mathbf{X}_{t}-\hat{\mathbf{X}}_{t \mid N}\right)\left(\mathbf{X}_{t}-\right.\right.$ $\left.\left.\hat{\mathbf{X}}_{t \mid N}\right)^{\mathrm{T}}\right]$ is the error covariance matrix when the information of the whole data series is used. In summary, the Kalman smoothing algorithm uses both the forward estimation result of the conventional Kalman filter and a backward filtering 

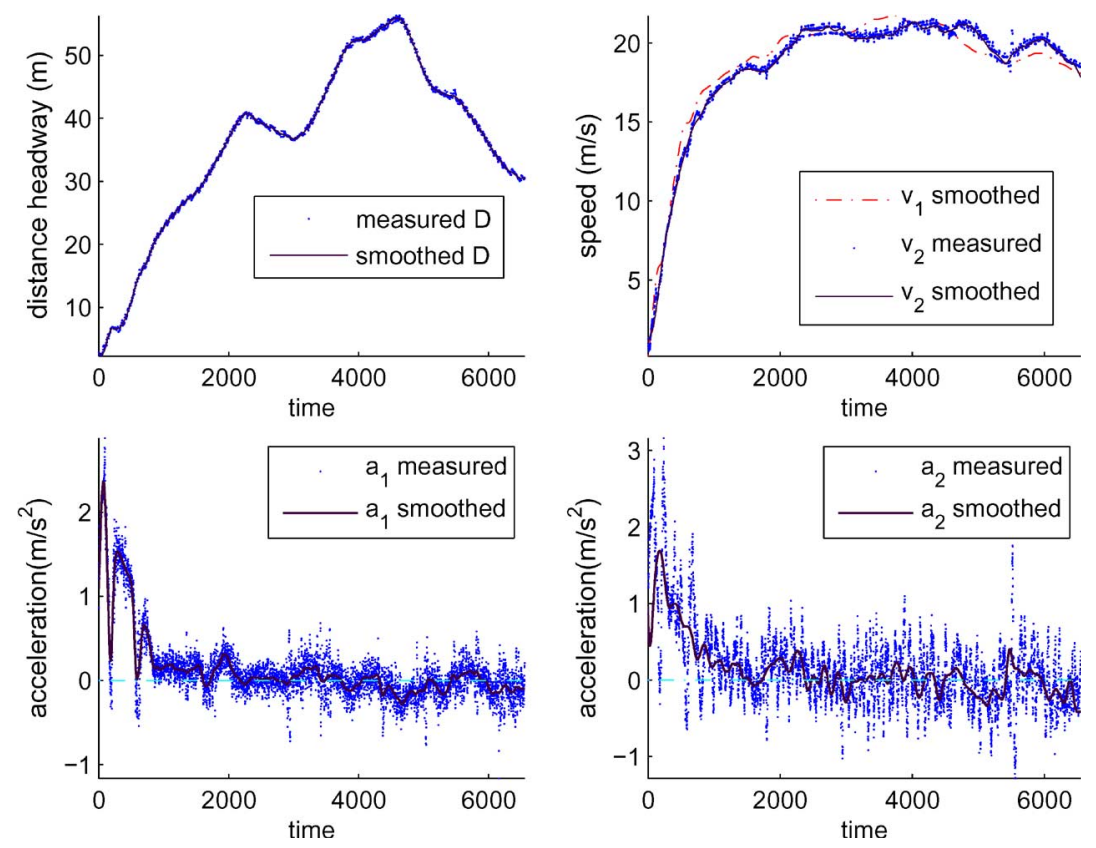

Fig. 6. Example of speed and acceleration profiles of both vehicles and the range between them before and after noise cancellation by the Kalman smoothing algorithm (the measurement time interval is $0.02 \mathrm{~s}$ ).

iteration. The final result should be, in principle, more reliable than that from the forward pass.

\section{Practical Results}

In practice, we have implemented and applied the Kalman smoothing algorithm to the logged data series of both the instrumented and observed vehicles. Thanks to the short time interval $h=0.02$ or the high measuring frequency $(f=50)$, the random walk model is applied for the acceleration time series of both vehicles, i.e., $\phi=1$ for (4). To cancel the noise by the Kalman smoother, the covariance matrices $R^{V}$ and $R^{W}$ for the noise processes need to be known. Noises are assumed to be white and uncorrelated to each other, and so, the two covariance matrices are diagonal. Hence, we only need to know the power levels, or variances, of the noises in both equations.

Time series of the instrumented car are smoothed at first. Noise for the position and speed is zero, and so, only the noise term in the random walk (4) needs to be determined. The equipment in the instrumented car can directly estimate the position and speed of the car, but the acceleration is derived from the speed data. Therefore, it is not necessary to include acceleration measurement in the observation equation, that is, $\mathbf{Y}(t)=\left[\hat{s}_{n}(t) \hat{v}_{n}(t)\right]^{\mathrm{T}}$, and

$$
H=\left(\begin{array}{lll}
1 & 0 & 0 \\
0 & 1 & 0
\end{array}\right)
$$

Since we do not know the noise in the observation data of traveling distance and speed, it becomes a filter design problem in which we have to choose appropriate noise covariance matrices to realize a tradeoff between tracking ability and noise rejection capability of the filter. According to the study in [14], the performance of the filter is mainly determined by the ratio of the covariance matrices. By adjusting the ratio, we can obtain more reasonable estimation of state profiles. In fact, the measurement noise in the speed and position time series of the instrumented car are rather small in comparison with the disturbance to the time series of the observed objects, making estimated results vary only within a small range.

Next, we apply the smoothing algorithm to cancel noise in the states of the observed car. As we have discussed in advance, the relative speed and acceleration in the measurement output of the following car are in fact derived from the distance measurement from laser beam scanning, and an online adaptive filter is applied to smooth data. Therefore, only the space headway measurement shall be adopted in our Kalman smoother since online estimation of relative speed and acceleration cannot improve our estimation. Then, the measurement equation becomes a first-order form of the general equation (6), where $\mathbf{Y}(t)=\hat{s}_{n}(t)$, and $H=\left[\begin{array}{lll}1 & 0 & 0\end{array}\right]$. As far as is known, noise for velocity and position in the state update equation are zero, but we give them a very small value to avoid the singular matrix. The key becomes determining the ratio between the power of the measurement noise for distance and that of the noise term in the random walk model of (4). Nevertheless, the power level of the noise term for the acceleration in the state equation can be estimated by computing the variance of the process $\theta(t)=a_{n-1}(t)-a_{n-1}(t-1)$, where $a_{n-1}(t)$ is the acceleration process of the instrumented vehicle. The noise in the range measurement is approximately known from the producer, and so, the parameters of the Kalman smoother can be tested out with small adjustments.

Remarkable changes of the noise level in the range measurement may appear when the time series includes a wide distance value, e.g., from 0 to $100 \mathrm{~m}$ (since the larger the range, the higher the measurement error). This indicates the nonstationary characteristic of the system, and smoothing different parts of the time series separately may give a better result than smoothing the whole time series at one stroke. Fig. 6 shows 

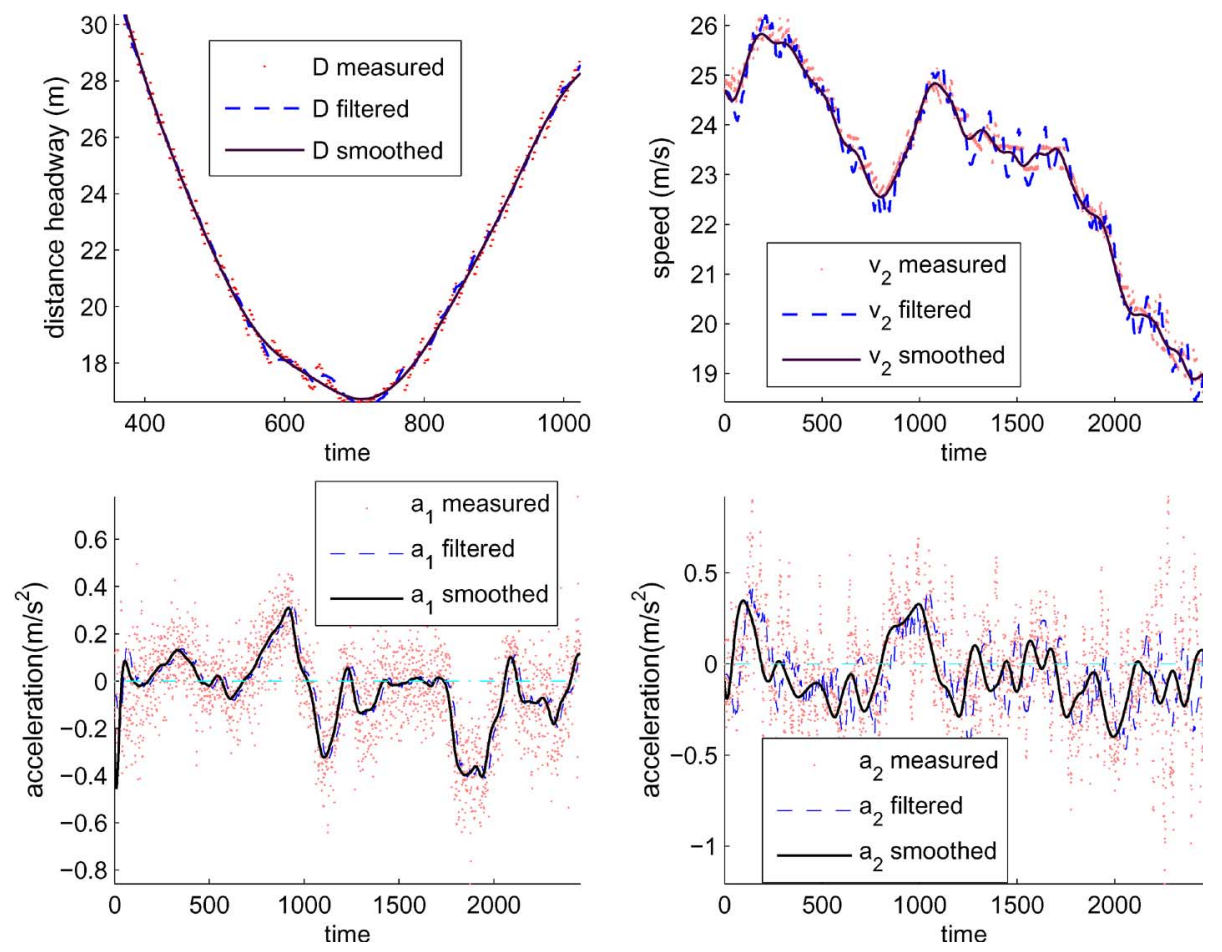

Fig. 7. Comparison example of estimation results from a car-following time series using the Kalman filter and Kalman smoother (the measurement time interval is $0.04 \mathrm{~s}$ ).

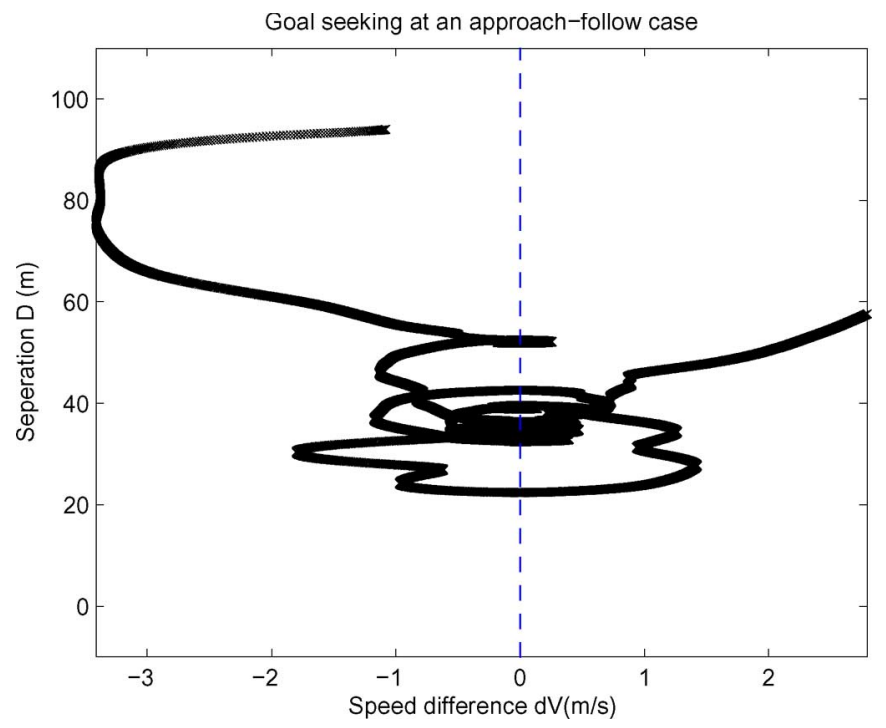

Fig. 8. Example of oscillation and goal seeking in a typical approach-follow case.

one of the results of the speed and acceleration profiles of the instrumented car and the observed car before and after applying the Kalman smoothing algorithm. Fig. 7 illustrates the difference between the estimation results from the Kalman filter and Kalman smoother. The Kalman smoother gives clearer state profiles for the following vehicle.

\section{Data Validity and Driver Properties}

After the data preprocessing, it is necessary to analyze the data patterns obtained. Wu et al. in [32] adopted a concept validation methodology in the comparison of their car-following model output with real data. The concepts used in this paper could also be used to check our data patterns. Accordingly, one of the important criteria is to appreciate that the separation at which the vehicle pair stabilizes is not actually constant but oscillates within a certain range due to the misperceptions in the decision process. This may cause overcompensation leading to goal seeking [32]. Fig. 8 shows a typical example, from our dataset, of oscillation and goal seeking behavior in the "approach-follow" case. In fact, the oscillation and goal-seeking phenomenon appears not only in this case but also in the "acceleration-follow" and "cut-in-follow" processes. We have examined the concept in most of our data patterns. 
Different drivers may have variations in those profiles due to their sensitivity differences in speed and distance or separation.

Besides surveys of data at a concept level, system identification methods [16] were also applied in our in-depth carfollowing data analysis. In our former study [25], the spectrum of all the time series were plotted, and the transfer functions between all observation inputs and the acceleration output were estimated. It was discovered that all these data series were narrowbanded, and the amplitude of the transfer functions showed bandpass properties, which agreed with the expectation that "human sensors" have bandpass properties. Moreover, it was easy to find the phase shift phenomenon in the phase plots of the transfer functions, and this indicates that drivers have reaction delays during the driving task. In the former study [25], we also showed that both the phase spectrum and coherence spectrum methods could be applied in driver reaction time estimation. However, they are limited in estimation accuracy due to the common spectral leakage problems [13] and rarity of validation methods.

\section{Classification of Car-Following Regimes}

It is widely known that driver behavior, especially car following, shows a property of regime adaptation due to the sensitivity thresholds of human beings. Therefore, drivers exhibit distinct characteristics in each regime of car-following behavior. This psychological concept has been introduced by modelers in building more realistic car-following models. In fact, two popular microscopic traffic simulation software (VISSIM and PARAMICS) both adopt car-following models with definitions of multiple regimes, although the classification of the regimes are different (see [9] and [21]). One common disadvantage of these models is that the regimes or thresholds are solely defined by the knowledge or commonsense of modelers, possibly with bias. Even though later researchers tried to calibrate some psychological thresholds by real data [32], a consolidated method has not been developed to identify the regime classifications based on properties in real data. One possible approach is to use supervised classification methods [31], e.g., Bayesian classification, but it requires detailed prior knowledge in different car-following regimes, e.g., probability distributions of the variables. In this section, we resort to unsupervised methods to identify the internal structure of the acquired data and separate the car-following data into several common regimes: acceleration, stable following, braking, approaching, and opening.

\section{A. Classical Unsupervised Clustering Algorithms}

$\mathrm{K}$-means clustering, also known as ISODATA [1], is a widely used unsupervised clustering algorithm to attribute multidimensional data into different clusters according to certain dissimilarity measures. In this method, the cluster number has to be determined at first, and each data point can be either a member of a cluster (membership degree $u$ is 1) or not a member of a cluster (membership degree $u$ is 0 ). By randomly determining the initial cluster centers, the algorithm searches iteratively the optimal combination of the membership degree matrix and cluster centers in order to minimize the summation of dissimilarity measures of data vectors to its cluster center, that is

$$
\mathbf{J}=\sum_{i=1}^{C} \sum_{k, k \in C_{i}} \mathbf{D}\left(\mathbf{x}_{\mathbf{k}}, \mathbf{c}_{\mathbf{i}}\right)
$$

where $\mathbf{x}_{\mathbf{k}}$ is the $k$ th data vector, and $\mathbf{c}_{\mathbf{i}}$ is the cluster center of $i$ th cluster $C_{i} . \mathbf{D}(\cdot, \cdot)$ is the measure of dissimilarity, and the Euclidean distance $\mathbf{D}\left(\mathbf{x}_{\mathbf{k}}, \mathbf{c}_{\mathbf{i}}\right)=d_{i k}^{2}=\left\|\mathbf{x}_{\mathbf{k}}-\mathbf{c}_{\mathbf{i}}\right\|^{2}$ is one of the ones applied the most.

Fuzzy C-means clustering [3] generalizes the K-means algorithm with adoption of the fuzzy membership degree. That is, each data vector can be attributed to several clusters with certain membership degrees between 0 and 1 , but the summation of the membership degrees for each data vector must be 1 . Hence, the objective function becomes

$$
\mathbf{J}=\sum_{i=1}^{C} \sum_{k=1}^{N} u_{i k}^{m} d_{i k}^{2}
$$

with the constraint

$$
\sum_{i=1}^{C} u_{i k}=1, \quad \forall i \in[1, N] .
$$

Solving the optimization problem leads us to the fuzzy C-means algorithm in the following.

1) Initialize a random membership matrix $U$ with (12) fulfilled.

2) Calculate $C$ cluster centers $\mathbf{c}_{\mathbf{i}}$ for $i=1, \ldots, C$ using

$$
\mathbf{c}_{\mathbf{i}}=\frac{\sum_{k=1}^{N} u_{i k}^{m} \mathbf{x}_{\mathbf{k}}}{\sum_{k=1}^{N} u_{i k}^{m}} .
$$

3) Compute the objective function and check whether the reduction is below a certain threshold.

4) Update the membership matrix $U$ using

$$
u_{i k}=\frac{d_{i k}^{-2 /(m-1)}}{\sum_{c=1}^{C}\left(d_{c k}\right)^{-2 /(m-1)}}
$$

and go to step 2).

An important question in both clustering algorithms is how to determine the number of clusters before application of them. The David-Bouldin index [8] is a classical way to determine the optimal clustering number. In many real-world applications, however, domain knowledge plays an essential role in the determination of this number, though it is sometimes necessary to use visualization tools.

\section{B. Fuzzy Clustering Algorithm With Time Continuity}

A common weakness of $\mathrm{K}$-means and fuzzy C-means algorithms is that both methods exploit the homogeneity of data in the multidimensional feature space without incorporating the inherent ordering of the data. In our case, the collected driving data are all time series. Therefore, a clustering algorithm with 
consideration of the time continuity of the data should be applied in our car-following regime classification. Initial ideas on fuzzy clustering considering spatial continuity were introduced in the area of image processing [22], [27]. Clustering of multivariate time series is sort of different from the image processing application, although the common principle reformulates the objective function of (11) with a modified dissimilarity measure based on each data point and its neighborhood and a penalty term discouraging sudden oscillations in the membership functions. In our application, a more general objective function is defined as follows:

$$
\mathbf{J}_{\mathbf{n}}=\sum_{i=1}^{C} \sum_{t=1}^{N} u_{i t}^{m} d_{i t}^{2}+\alpha \sum_{i=1}^{C} \sum_{t=1}^{N} u_{i t}^{m} \sum_{\delta t=-l, \delta t \neq 0}^{l} \Phi_{\delta t} R_{i t, \delta t}
$$

with the constraint

$$
\sum_{i=1}^{C} u_{i t}=1 \quad \forall t \in[1, N]
$$

where

$$
R_{i t, \delta t}=\sum_{p=1, p \neq i}^{C} u_{p, t+\delta t}^{m}
$$

is the summation of the $m$ exponent of the membership degrees that the neighbor point at $t+\delta t$ belongs to all clusters except cluster $i ; \alpha$ represents the impact factor of the regularization term, and the function $\Phi_{\delta t}$ describes the contribution weight from the data point's neighborhood, which can be defined based on, e.g., local correlations information of the time series. By introducing this additional term, we can restrict the case that a data vector belongs to a certain class with a high degree, but its neighbors have a high membership degree summation to other classes (vice versa). Therefore, the final membership function will be somehow regularized. Based on the derivations (showed in the Appendix), we can still apply the procedures of the fuzzy C-means algorithm but replace (14) with

$$
u_{i t}=\frac{\left(d_{i t}^{2}+2 \alpha \sum_{\delta t=-l, \delta t \neq 0}^{l} \Phi_{\delta t} R_{i t, \delta t}\right)^{-1 /(m-1)}}{\sum_{c=1}^{C}\left(d_{c t}^{2}+2 \alpha \sum_{\delta t=-l, \delta t \neq 0}^{l} \Phi_{\delta t} R_{c t, \delta t}\right)^{-1 /(m-1)}} .
$$

\section{Implementation and Numerical Experiments}

Fuzzy clustering technology has been introduced in the classification of traffic regimes and driver-behavior patterns in some ITS applications. However, few studies considered equalizing the dimensional scale of data vectors before applying a clustering algorithm. This leads to a consequence that vectors with larger numerical values will be actually more preponderant during the regime classification. For example, in our case, distance headway (probably with speed of the following car) will determine the final regimes if we do not equalize the scale in advance. Hence, the resulting clusters and boundaries may not reflect the internal structure of the multidimensional data vectors. In this application, we consider two scaling methods; the first method scale multidimensional data

$$
\left[\begin{array}{lllll}
D(t) & d v(t) & v_{2}(t) & a_{1}(t) & a_{2}(t)
\end{array}\right]^{\mathrm{T}}
$$

to multivariate time series with entry values between 0 and 1 by applying

$$
\hat{x}(t)=\frac{x(t)-\phi_{x}}{\psi_{x}-\phi_{x}}
$$

where $x(t)$ is equal to each of $D(t), d v(t), v_{2}(t), a_{1}(t)$, and $a_{2}(t), \phi_{x}=\min \{x(t)\}$, and $\psi_{x}=\max \{x(t)\}$; the second method normalizes the dataset by applying

$$
\hat{x}(t)=\frac{x(t)-m_{x}}{\sigma_{x}}
$$

where $m_{x}=\mathbf{E}[x(t)]$, and $\sigma_{x}$ is the standard deviation of the time series $x(t)$. The first scaling method hypothesizes superspherical clusters (that is, the vectors in each dimension are equally important), whereas the second one assumes normal distribution of the data population and considers the statistical properties (variance) of the time series. Both methods are options for our car-following data analysis. After scaling of the data, possible cluster (regime) numbers are determined from video images based on our prior understanding on each carfollowing pattern, and one proper cluster number is chosen. The classical fuzzy C-means method is first applied to see if car-follow regimes can be classified from the dataset in an understandable manner. Fig. 9 shows an example that a vehicle accelerated from a stop behind the leading car and then entered the stable following region. This procedure is qualitatively obvious according to the video record, but the boundary is difficult to determine. By applying the fuzzy C-means algorithm, we can classify the data into two continuous regimes and determine the boundary. However, fuzzy C-means method sometimes cannot classify the continuous regimes successfully since it assumes the independence between data points. Therefore, application of consolidated fuzzy C-means method is necessary. Fig. 10 shows an example where a vehicle followed a leading car after merging and then they were separated (opening). The classical clustering method cannot completely partition data into two continuous regimes because the belongingness of the middle part is vague. This can be solved by the proposed consolidated fuzzy C-means method, and it strengthens a remarkable boundary while unraveling the membership fuzziness between those two regimes. In another case study, the method is evaluated in a more complicated situation where the regimes are not so clear, even in the video recording. In this example, a vehicle first approached, then followed remotely behind the leading car, and then, two vehicles were separated completely. The classical fuzzy C-means method cannot solve this puzzle (on the left of Fig. 11), but the consolidated fuzzy C-means algorithm does propose a solution: Cluster 1 is the approaching regime, cluster 2 belongs to the following case, and cluster 3 can be attributed to unaffected separation or opening. 


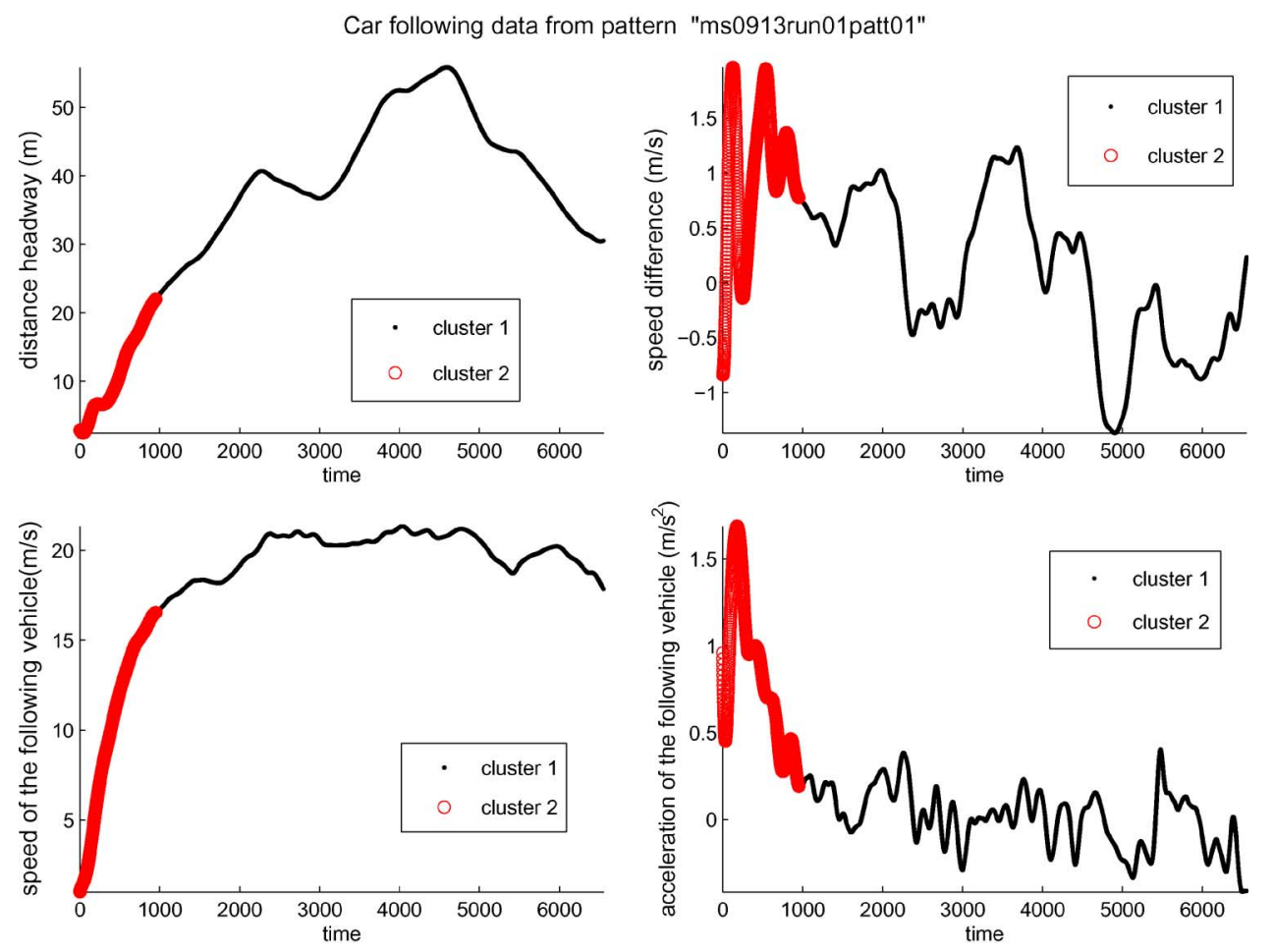

Fig. 9. Example of successful classification of a following regime (cluster 1) and an acceleration regime (cluster 2) in a car-following time series using fuzzy C-means algorithm $(m=2$ and the measurement time interval is $0.04 \mathrm{~s})$.
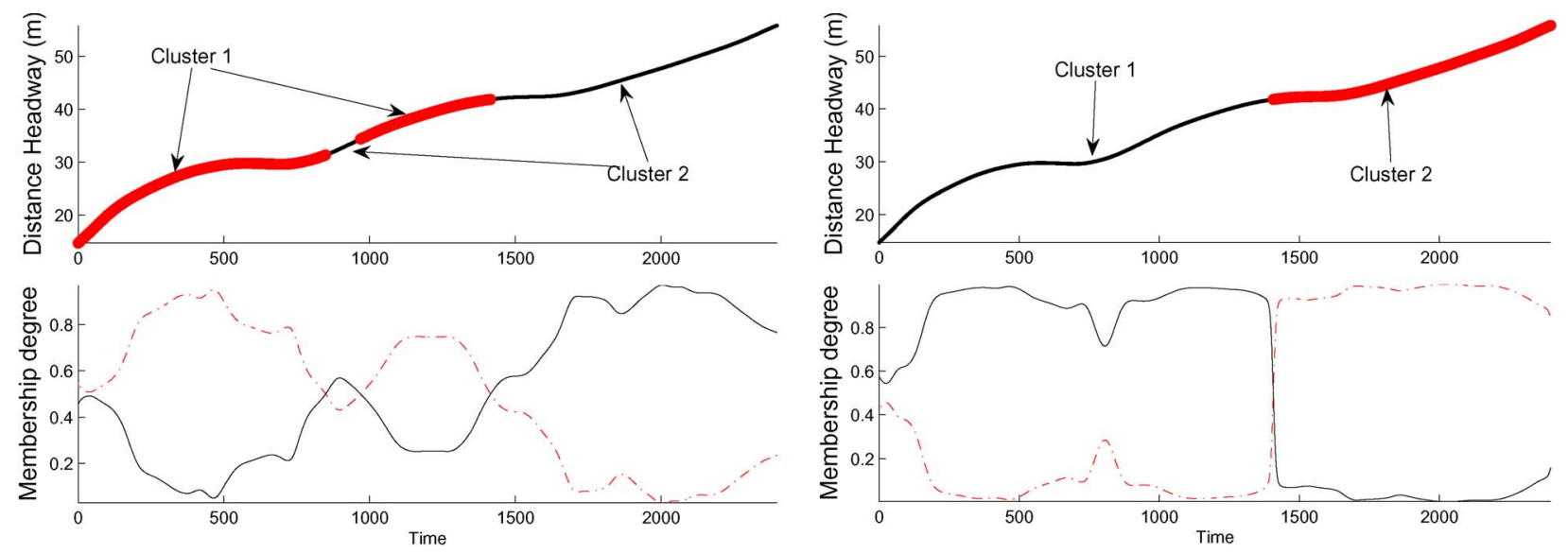

Fig. 10. Comparison result of classifying a following regime (after merging) and an opening regime. (Left) Result using classical fuzzy C-means algorithm with the distance headway attributing to each cluster (upper) and the membership function of each regime (lower) $(m=2)$. (Right) Result using consolidated fuzzy C-means algorithm with the distance headway attributing to each cluster (upper) and the membership function of each regime (lower) $(m=2, \beta=2 \alpha=30$, and $l=15$ ).

It is noticeable that values of the regulation factor and number of neighbors adopted in the objective function directly affect our final results. An appropriate regulation factor is often determined by cross-validation, that is, a small part of the dataset is used to evaluate which factor gives the least dissimilarity summation within that data population, and then, the regulation factor is applied for clustering of the whole dataset. This approach is reported to be successful in the spacial data analysis [27]. In our study of car-following time series, cross-validation is not practically applicable. Meanwhile, the objective is to partition multivariate time series data into continuous regimes by applying the regulation term. Therefore, the regulation factor does not necessarily optimize the objective function. An innovation in this clustering method is the consideration of the neighborhood in the regulation term. In theory, a larger regulation factor often makes it more difficult to optimize the objective function, and the fuzzy clustering algorithm does not ensure capturing the global optimum. Averaging the regulation term by considering the neighborhood with local weights can smooth the final objective function and then reduce the chance of trapping into local minimum. In this application, we define the local weight function $\Phi_{\delta t}$ as a simple triangular function, i.e., the weight decreases linearly and vanishes after an interval according to, e.g., the autocorrelation. 

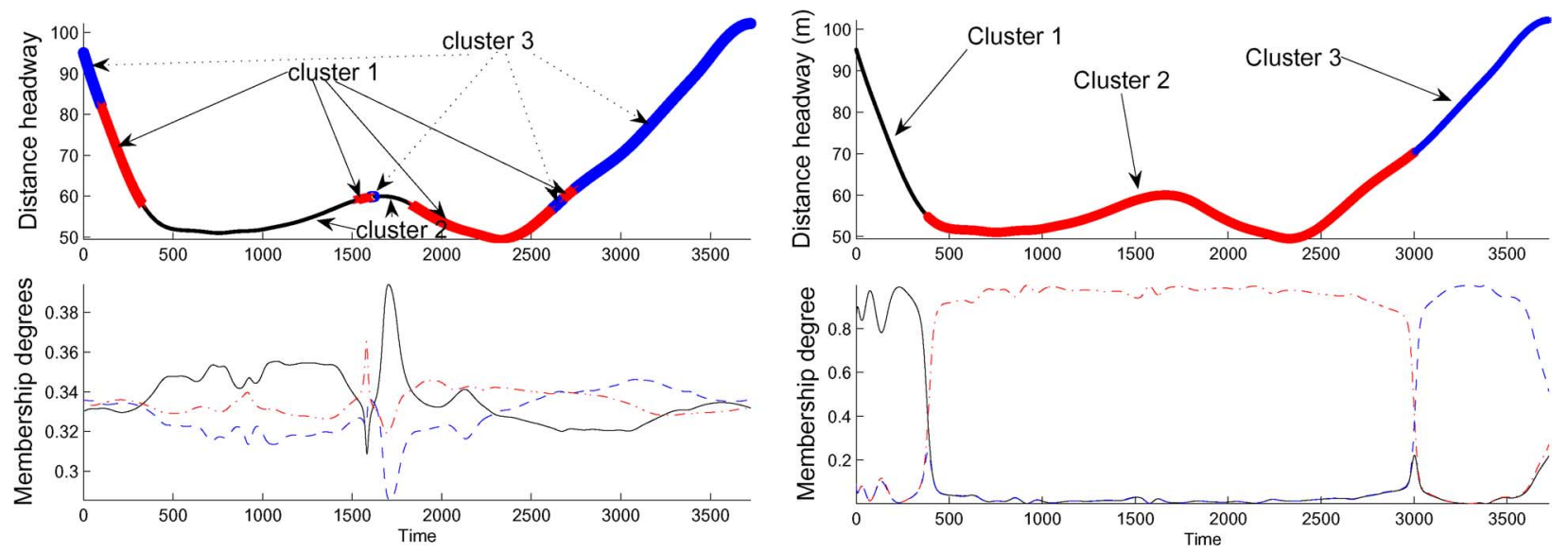

Fig. 11. Comparison result of classifying a car-following time series with vague regimes. (Left) Result using classical fuzzy C-means algorithm with the distance headway attributing to each cluster (upper) and the membership function of each regime (lower) $(m=2)$. (Right) Result using the consolidated fuzzy C-means algorithm with the distance headway attributing to each cluster (upper) and the membership function of each regime (lower) $(m=2, \beta=2 \alpha=100$, and $l=30)$.

In general, the proposed algorithm has been successfully applied in regime classification of our collected car-following time series whenever the fuzzy C-means algorithm gives us confusing results. The classification by this algorithm clarifies the boundaries between different car-following regimes and proves our prior knowledge in those regimes in many cases. However, the method inherits a common problem in the classical fuzzy C-means algorithm [15]: It does not ensure finding the global optimum point under a certain regulation factor, and the algorithm may converge to a local minimum point, depending on the initial random guess of the cluster centers. A further improvement of the method may apply evolutionary algorithms to find the global optimum as well as to optimize the scale factors in (19) and (20). In addition, this algorithm is computationally expensive in comparison with classical fuzzy C-means algorithm. To conduct extensive numerical experiments, we implemented the consolidated fuzzy $\mathrm{C}$-means algorithm using the $\mathrm{C} / \mathrm{C}++$ language and compiled it as a module, which could be called from the MATLAB/MEX platform.

\section{Summary AND Further Research}

In this research, we employed an instrumented vehicle with modern ITS features to acquire driver-behavior patterns in real traffic. Car-following behavior data of randomly observed drivers were captured for further model calibration and validation in traffic simulation. The data could also be used in the development of ACC systems. To eliminate measurement noise, the Kalman smoothing algorithm, which is an extension of the conventional Kalman filter, was applied to preprocess the measured data. The smoothed result shows clear car-following patterns and has been applied in our model calibration study. Meanwhile, to classify different car-following regimes, we developed a robust fuzzy clustering algorithm in which time continuity in the acquired dataset was considered. The application results showed that the method could give a more reliable classification of car-following regimes based on the regime numbers determined by video analysis. In fact, this method could also be utilized in the identification of the psychophysical thresholds from driver-behavior data, but we have not applied for that purpose in our current research. This paper has reported three main procedures in our driver-behavior (mainly car following) study: data acquisition experiment, data preprocessing, and car-following pattern classification. Further analysis of car-following behavior in different regimes is still ongoing. Meanwhile, new functionalities on the vision ability of the instrumented vehicle are necessary for accurately collecting and efficiently analyzing more car-following data and for extending our study on lane-changing behavior.

\section{APPENDIX}

To derive an iterative algorithm for evaluation of the clustering centers and membership matrix, a zero gradient condition can be applied with fixed values on $m, C$, and $\alpha$. Using the Lagrange multipliers to enforce the constraints in (12), we reformulate the objective function as

$$
\begin{array}{r}
\mathbf{J}_{\mathbf{n}}^{\prime}=\sum_{i=1}^{C} \sum_{t=1}^{N} u_{i t}^{m} d_{i t}^{2}+\alpha \sum_{i=1}^{C} \sum_{t=1}^{N} u_{i t}^{m} \sum_{\delta t=-l, \delta t \neq 0}^{l} \Phi_{\delta t} R_{i t, \delta t} \\
+\sum_{t=1}^{N} \lambda_{t}\left(1-\sum_{i=1}^{C} u_{i t}\right) .
\end{array}
$$

Assuming the cluster centers $\mathbf{c}_{\mathbf{i}}$ are fixed, we fulfill the zero condition to the partial derivative with respect to $u_{i t}$

$$
\begin{aligned}
\frac{\partial \mathbf{J}_{\mathbf{n}}^{\prime}}{\partial u_{i t}}=\frac{\partial}{\partial u_{i t}}\left(\sum_{i=1}^{C} \sum_{t=1}^{N} u_{i t}^{m} d_{i t}^{2}+\alpha \sum_{i=1}^{C} \sum_{t=1}^{N} u_{i t}^{m} \sum_{\delta t=-l, \delta t \neq 0}^{l} \Phi_{\delta t} R_{i t, \delta t}\right. \\
\\
\left.\quad+\sum_{t=1}^{N} \lambda_{t}\left(1-\sum_{i=1}^{C} u_{i t}\right)\right) \\
=m u_{i t}^{m-1}\left(d_{i t}^{2}+2 \alpha \sum_{\delta t=-l, \delta t \neq 0}^{l} \Phi_{\delta t} R_{i t, \delta t}\right)-\lambda_{t} \\
=0 .
\end{aligned}
$$


Therefore, the following equation can be derived:

$$
u_{i t}=\left(\frac{m\left(d_{i t}^{2}+2 \alpha \sum_{\delta t=-l, \delta t \neq 0}^{l} \Phi_{\delta t} R_{i t, \delta t}\right)}{\lambda_{t}}\right)^{-1 /(m-1)}
$$

and by applying the constraints, we get

$$
\begin{aligned}
\sum_{i=1}^{C} u_{i t} & =1 \\
& =\sum_{i=1}^{C}\left(\frac{m\left(d_{i t}^{2}+2 \alpha \sum_{\delta t=-l, \delta t \neq 0}^{l} \Phi_{\delta t} R_{i t, \delta t}\right)}{\lambda_{t}}\right)^{\frac{-1}{(m-1)}} .
\end{aligned}
$$

Therefore, the following necessary condition can be obtained by combining the equations above:

$$
u_{i t}=\frac{\left(d_{i t}^{2}+2 \alpha \sum_{\delta t=-l, \delta t \neq 0}^{l} \Phi_{\delta t} R_{i t, \delta t}\right)^{-1 /(m-1)}}{\sum_{c=1}^{C}\left(d_{c t}^{2}+2 \alpha \sum_{\delta t=-l, \delta t \neq 0}^{l} \Phi_{\delta t} R_{c t, \delta t}\right)^{-1 /(m-1)}} .
$$

Next, by fixing the membership matrix $U$ or $u_{i t}$, the necessary condition on the cluster centers to minimize the objective function is identical to the fuzzy C-means algorithm

$$
\mathbf{c}_{\mathbf{i}}=\frac{\sum_{k=1}^{N} u_{i k}^{m} \mathbf{x}_{\mathbf{k}}}{\sum_{k=1}^{N} u_{i k}^{m}}
$$

because the additional term in our objective function is not a function of the cluster centers $\mathbf{c}_{\mathbf{i}}$. The derivation is similar to that in the original development of the fuzzy C-means algorithm [3].

\section{ACKNOWLEDGMENT}

The authors would like to thank Prof. K.-L. Bång at the Department of Transportation and Logistics for his support on data collection using the advanced instrumented vehicle, developed by Volvo Technology and belonging to the Division of Transportation and Logistics (TOL), Department of Transport and Economics, KTH, and his helpful advice on the experiment design and research methodology. The authors would also like to thank the researchers at Volvo Technology for sharing their expert knowledge on the equipment.

\section{REFERENCES}

[1] G. H. Ball and D. J. Hall, "A clustering technique for summarizing multivariate data," Behav. Sci., vol. 12, no. 2, pp. 153-155, Mar. 1967.

[2] J. Bengtsson, "Adaptive cruise control and driver modeling," Ph.D. dissertation, Dept. Autom. Control, Lund Inst. Technol., Lund, Sweden, Dec. 2001
[3] J. C. Bezdek, Pattern Recognition With Fuzzy Objective Function Algorithms. New York: Plenum, 1981.

[4] M. Brackstone and M. McDonald, "Dynamic behavioural data collection using an instrumented vehicle," Transp. Res. Rec., vol. 1689, pp. 9-17, 1999.

[5] P. J. Brockwell and R. A. Davis, Introduction to Time Series and Forecasting. New York: Springer-Verlag, 1996.

[6] P. Chakroborty and S. Kikuchi, "Evaluation of general motors based car following models and a proposed fuzzy inference model," Transp. Res. Part C, vol. 7, no. 4, pp. 209-235, Aug. 1999.

[7] R. E. Chandler, R. Herman, and E. W. Montroll, "Traffic dynamics: Studies in car following," Oper. Res., vol. 6, no. 2, pp. 165-184, 1958.

[8] D. L. Davies and D. W. Bouldin, "A cluster separation measure," IEEE Trans. Pattern Anal. Mach. Intell., vol. PAMI-1, no. 4, pp. 224-227, Apr. 1979.

[9] H. T. Fritzsche, "A model for traffic simulation," Transp. Eng. Contrib. vol. 5, pp. 317-321, 1994.

[10] D. C. Gazis, R. Herman, and R. W. Rothery, "Nonlinear follow-the-leader models of traffic flow," Oper. Res., vol. 9, no. 4, pp. 545-567, 1961.

[11] M. S. Grewal and A. P. Andrews, Kalman Filtering: Theory and Practice. Englewood Cliffs, NJ: Prentice-Hall, 1993.

[12] S. Hakin, Kalman Filtering and Neural Networks. New York: Wiley, 2001.

[13] M. H. Hayes, Statistical Digital Signal Processing and Modeling. Toronto, ON, Canada: Wiley, 1996.

[14] H. Hjalmarsson and B. Ottersten, Lecture Notes in Adaptive Signal Processing. Stockholm, Sweden: Royal Inst. Technol., 2002.

[15] C.-T. Sun, J.-S. Jang, and E. Mizutani, Neuro-Fuzzy And Soft Computing: A Computational Approach to Learning and Machine Intelligence. Englewood Cliffs, NJ: Prentice-Hall, 1997.

[16] R. Johansson, System Modeling and Identification. Englewood Cliffs, NJ: Prentice-Hall, 1993.

[17] R. E. Kalman, "A new approach to linear filtering and prediction problems," Trans. ASME J. Basic Eng. D, vol. 83, no. 1, pp. 35-45, Mar. 1960.

[18] S. M. Kay, Fundamentals of Statistical Signal Processing: Estimation Theory. Englewood Cliffs, NJ: Prentice-Hall, 1993.

[19] T. Kim, D. J. Lovell, and Y. Park, "Limitation of previous models on car-following behaviours and research needs," in Proc. 82th TRB Annu. Meeting, Washington, D.C., 2003.

[20] I. Kosonen, A. Gutowski, F. Davidsson, T. Sauerwein, P. Blad, and D. Aalto, "Traffic Performance on Major Arterials," Center for Traffic Simulation Research, Royal Inst. Technol., Stockholm, Sweden, 2001. Final Rep. Part (b-f).

[21] W. Leutzbach and R. Wiedemann, "Development and applications of traffic simulation models at the Karlsruhe Institut fur Verkehrwesen," Traffic Eng. Control, vol. 27, pp. 270-278, May 1986.

[22] A. W. C. Liew, S. H. Leung, and W. H. Lau, "Fuzzy image clustering incorporating spatial continuity," Proc. Inst. Electr. Eng.-Vis. Image Signal Process., vol. 147, no. 2, pp. 185-192, Apr. 2000.

[23] X. Ma, "Report on the development of TPMA. 1.0: A manual for the developers," Center for Traffic Simulation Res., Royal Inst. Technol., Stockholm, Sweden, Mar. 2005. Internal Rep. (Draft).

[24] X. Ma and I. Andréasson, "Predicting the effects of ISA penetration grades on pedestrian safety by simulation," Accident Anal. Prev., vol. 37, no. 6, pp. 1162-1169, Nov. 2005.

[25] - "Driver reaction time estimation from the real car following data and its application in GM-type model evaluation," in Transp. Res. Rec., 2006 , in press.

[26] X. Ma, L. Engelsson, I. Andréasson, and G. Lind, "Evaluation of safety effects of various ISA vehicle penetration grades by microscopic simulations," Center for Traffic Simulation Res., Royal Inst. Technol., Stockholm, Sweden, Tech. Rep. CTR2004:14, Feb. 2004.

[27] D. L. Pham, "Spatial models for fuzzy clustering," Comput. Vis. Image Underst., vol. 84, no. 22, pp. 285-297, Nov. 2001.

[28] J. Rasmussen, Information Processing and Human-Machine Interaction: An Approach to Cognitive Engineering. New York: Elsevier, 1986.

[29] H. W. Sorenson, Kalman Filtering: Theory and Applications. Piscataway, NJ: IEEE Press, 1985.

[30] M. A. P. Taylor and W. Young, Traffic Analysis. Melbourne, Australia: Hargreen, 1988.

[31] S. Theodoridis and K. Koutroumbas, Pattern Recognition. San Diego, CA: Academic, 1999.

[32] J. P. Wu, M. Brackstone, and M. McDonald, "The validation of a microscopic simulation model: A methodological case study," Transp. Res. Part $C$, vol. 11 , no. 6 , pp. 463-479, 2003. 


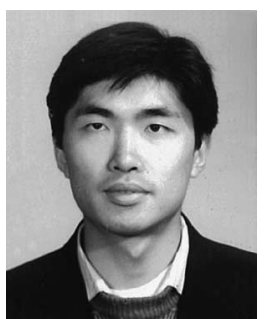

Xiaoliang Ma (S'06) received the B.E. degree in civil engineering from Beijing University of Technology, Beijing, China, in 1994 and the M.S. degree in applied mathematics from Royal Institute of Technology (KTH), Stockholm, Sweden, in 2002. He is currently working toward the Ph.D. degree at the Center for Traffic Simulation Research (CTR), KTH.

Now he is working toward the CTR as a Research Assistant. His main research interests include modeling and simulation of complex behavior and systems, especially driver behavior and vehicle-based intelligent transportation system (ITS) modeling and computational intelligence, including neural networks, fuzzy systems, and hybrid modeling. He worked with finite-element (FE) computing at the material physics group, Swedish Paper Research Institute, from 2001 to 2002, and was a System Analyzer and Software Developer with Frost \& Sullivan in 1998 and 1999.

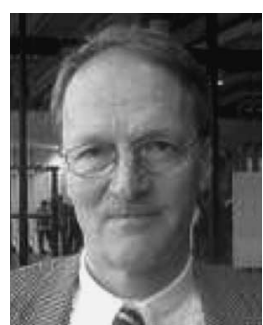

Ingmar Andréasson received the Ph.D. degree in applied mathematics from Royal Institute of Technology (KTH), Stockholm, Sweden, in 1972.

$\mathrm{He}$ is an Adjunct Professor of transport and logistics and, since 1997, has been the Director for the Center for Traffic Simulation Research at KTH. Before that, he directed the Advanced Transport Systems Program at Chalmers University of Technology, Göteborg, Sweden. Between 1973 and 1988, he was President and Chairman of Volvo Transportation Systems. He is Cofounder of LogistikCentrum, offering consultancy and research in transport and logistics. He is the author of a textbook on simulation methodology and has published numerous contributions in simulation modeling and transport analysis and planning. His recent research interests include modeling of driver-behavior and traffic performance in congested road networks and Personal Rapid Transit systems.

Dr. Andréasson is a committee member of the U.S. Transportation Research Board (TRB) and a board member of the Advanced Transit Association (ATRA). 